\title{
Study on Optimization of Extraction Technology of Anthocyanin Extracted From Purple Eggplant Peel by Quadric Orthogonal Rotation Combination Design
}

\author{
Xue Wang, Taifan Sun*, Shuting Zhao, Jinqiu Ji, Jiaqi Li, Yaowen Xing Xiaojing Zhou \\ College of Science, Heilongjiang Bayi Agricultural University
}

\begin{tabular}{ll}
\hline DOI: $10.36348 /$ sijcms.2019.v02i08.002 & | Received: 14.12 .2019 | Accepted: 22.12 .2019 | Published: 26.12 .2019
\end{tabular}

*Corresponding author: Taifan Sun

\section{Abstract}

In order to obtain the optimal extraction conditions of anthocyanin in purple eggplant peel, purple eggplant peel was used as experimental material, the single factor test conditions were screened through determinating the content of anthocyanin in the crude extraction solution. The optimum range of the concentration of ethanol, the ratio of liquid and material ratio, the time of extraction, and the temperature of extraction were determined. Then the quadratic regression orthogonal rotation combination design was used to optimize the extraction process of anthocyanin. Four factors and regression model of content of anthocyanin were obtained. The results showed that the factors affecting the extraction efficiency of anthocyanins were as follows: the concentration of ethanol $>$ the temperature of extraction $>$ the ratio of liquid and material ratio $>$ the time of extraction. Finally, the optimum extraction conditions of anthocyanin extracted from purple eggplant peel were obtained as follows: the concentration of ethanol was $78.6 \%$, the ratio of liquid and material ratio was $60: 1\left(\mathrm{~mL} \mathrm{~g}^{-1}\right)$, the temperature of extraction was $20^{\circ} \mathrm{C}$, the time of extraction was $150 \mathrm{~min}$, and the content of anthocyanin was up to 4.2399 (mg g-1). The crude extracting solution of anthocyanin was clear and stable, and the color of stable extracting solution was dark red. The validation experiment was carried out at the optimized conditions, and the preferable results were obtained, which showed that the optimization of extraction technology of anthocyanin in purple eggplant peel by this method was reliable, and which had practical guiding significance.

Keywords: purple eggplant peel; anthocyanin; orthogonal rotary combination design; extraction; regression model.

Copyright @ 2019: This is an open-access article distributed under the terms of the Creative Commons Attribution license which permits unrestricted use, distribution, and reproduction in any medium for non-commercial use (NonCommercial, or CC-BY-NC) provided the original author and source are credited.

\section{INTRODUCTION}

Anthocyanin is a kind of natural pigment, which widely found in plants, belongs to flavonoid [1] It is abundant in grapes, blueberries, blackcurrants, purple potatoes, black rice, cherries, blood oranges, mulberry, morsels and other plants. Generally, anthocyanin is formed by glycoside bonds between galactose and glucose [2], and it exist rarely in the form of free anthocyanins. Anthocyanin can not only make plants, flowers and fruits show gorgeous colors, but also have strong physiological activities, such as antioxidant, anti-aging, cancer prevention, cerebrovascular protection, improvement of eyesight, and the role of beauty[1], which are widely used in food, drugs, cosmetics and other fields. The extraction methods of anthocyanin mainly include organic solvent extraction, ultrasonic extraction, microwave assisted extraction, supercritical fluid extraction, enzyme extraction, etc[3-7]. With the improvement of people's living standard, people pay more and more attentions to food safety, and hold a cautious attitude towards synthetic pigments. That will promote the development and utilization of anthocyanins.

Eggplant, which has the characteristics of a wide planting area, richness in nutrients, loving by people, is a common vegetable. Eggplant is bitter and cold, and has many benefits, such as treating bruises and stopping bleeding, as well as treating cold and heat and reducing swelling and pain. At present, most studies on eggplant focus on the distribution of its resources, planting techniques and other aspects[8], and the research on anthocyanin which is an important bioactive ingredient in eggplant skin, is much less than that in blueberries and grapes[9]. On the basis of single factor extraction, the extraction process of anthocyanin from eggplant skin was optimized by quadratic orthogonal rotation combination design, which provided theoretical basis for further processing and comprehensive utilization of eggplant. 


\section{MATERIALS}

Experimental sample

Long eggplants were bought in the

supermarket.

\section{Reagents}

Centaurenin-3-O-glucoside

(chromatographical pure, 98\%), purchased from hefei bomei biotechnology co., LTD ; anhydrous ethanol, hydrochloric acid, sodium chloride, glacial acetic acid and anhydrous sodium acetate which were all analytically pure and purchased from Damao chemical reagent factory in Tianjin.

\section{Instruments}

Electronic analytical balance (ALC-310.3), which purchased from Shanghai precision scientific instrument co., LTD. Concentrated type constant temperature heating magnetic stirrer (DF-10S), which purchased from gongyi yuhua instrument co., LTD. Uvvisible spectrophotometer (T6), which purchased from Beijing general instrument co., LTD. Circulating water type multipurpose vacuum pump (SHB-IIIA), which purchased from zhengzhou Great Wall industry \&trade co., LTD. Acidity meter (LE438), which purchased mettler-Toledo instrument LTD.

\section{METHOD}

\section{The pretreatment of sample}

The fresh eggplant, which bought in the supermarket, washed and peeled firstly, the eggplant peel was dried and crushed secondly, and it was sealed and refrigerated for use at last.

\section{Determination of the maximum absorption wavelength}

Anthocyanin standard sample, Centauin-3-Oglucoside $(0.0010 \mathrm{~g})$, was weighed accurately, and placed in scale tube $(25.00 \mathrm{~mL})$, then it was dissolved by the ethanol solution $(70 \%, \mathrm{pH}=1.0)$, the volume of the solution was constant to $25.00 \mathrm{~mL}$, thus the standard solution $\left(40 \mathrm{ug} \cdot \mathrm{ml}^{-1}\right)$ was received. The absorbance of the solution was tested at 400-700 nm, and the maximum absorption wavelength was obtained. The absorbance of the sample solution was also tested at 400-700 nm.

\section{The calculation of anthocyanin content in purple eggplant peel}

$1.5000 \mathrm{~g}$ purple eggplant peel was put in the conical flask $(250 \mathrm{~mL})$, and then the solution was stired well after the acidic ethanol was added, the coarse solution was achieved after filtering. $1.00 \mathrm{~mL}$ coarse solution was put in the volumetric flask $(25.00 \mathrm{~mL})$, and then the buffer solution $(\mathrm{pH}=1.0)$ was added to the scale mark of $25.00 \mathrm{~mL}$, so the sample solution was obtained, the reference solution was obtained as the same. The absorbances of those two solutions was test at $530 \mathrm{~nm}$ and $700 \mathrm{~nm}$, and then $\mathrm{pH}$ differential method was used to calculate the content canthocyanins[10], the formula is as follows.

$$
\begin{aligned}
& \text { The content of anthocyanins }\left(\mathrm{mg} \cdot \mathrm{g}^{-1}\right) \\
& =(\Delta \mathrm{A} \times \mathrm{V} \times \mathrm{D} \times \mathrm{M}) /(\varepsilon \times \mathrm{m} \times \mathrm{b})
\end{aligned}
$$

The symbol $\Delta \mathrm{A}$ is the difference of absorbance, $\Delta \mathrm{A}=\left(\mathrm{A}_{530, \mathrm{pH} 1.0}-\mathrm{A}_{700, \mathrm{pH} 1.0}\right)-\left(\mathrm{A}_{530, \mathrm{pH} 4.5}-\right.$ $\left.\mathrm{A}_{700, \mathrm{pH} 4.5}\right), \mathrm{V}$ is the volume of coarse solution, $\mathrm{D}$ is the dilution multiple, $\mathrm{M}$ is the molar mass of cornstoin-3-oglucoside $\left(449.2 \mathrm{~g} \cdot \mathrm{mol}^{-1}\right), \varepsilon$ Is the molar absorption coefficient of cornstoin-3-o-glucoside $(26$ 900(L.mol$1 \cdot \mathrm{cm}-1)[10] ; \mathrm{m}$ is the quality of raw material, $\mathrm{b}$ is the thickness of colorimetric dish $(1 \mathrm{~cm})$.

\section{The test of single factor}

$1.5000 \mathrm{~g}$ purple eggplant peel was put in the conical flask $(250 \mathrm{~mL})$, the extraction was carried out by changing the concentration of ethanol, the mixture ratio, the extraction time, and the temperature, etc., and the optimal ranges of the four influencing factors were determined through calculating the content of the anthocyanin in the extraction solution.

\section{The experimental design of quadratic orthogonal rotation combination}

The appropriate factor range was selected through the single-factor test, and the design experiment of quadratic orthogonal rotating combination was carried out at the level of four factors and five. The anthocyanin content was used as the investigation index to obtain the optimal extraction process conditions [11].

\section{RESULTS AND ANALYSIS}

The determination of maximum absorption wavelength

Cornflowers-3-O-glucoside used as the standard was scanned at 400 700 $\mathrm{nm}$. The results were shown in figure 1. According to the absorption curve, the maximum absorption wavelength of the purple eggplant peel samples was at $530 \mathrm{~nm}$. Therefore, the anthocyanin content was determined at $530 \mathrm{~nm}$ in this study.

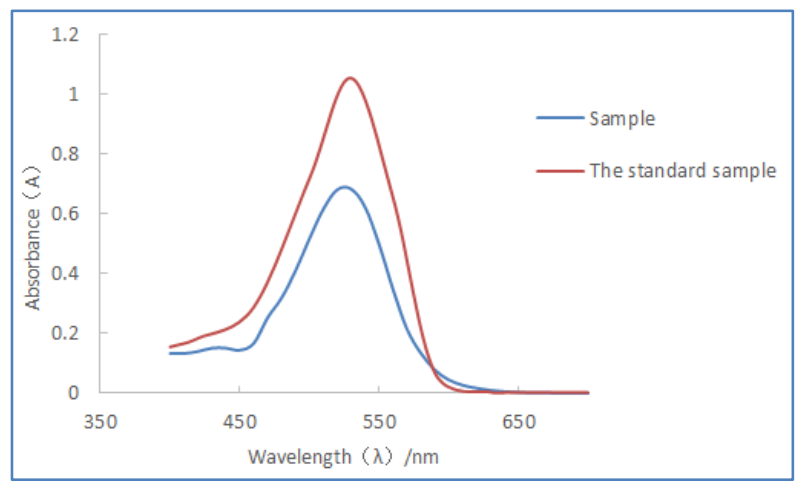

Fig-1: Ultraviolet-visible spectra of anthocyanins 


\section{The test of single factor}

\section{The influence of ethanol concentration}

Figure 2 shows the changes of anthocyanin contents according to the changing of ethanol concentrations. It can be seen that the concentration of ethanol has a great influence on the extraction of anthocyanins. When the concentration of extraction agent increases, the content of anthocyanins increases. The extraction effect is better when the concentration of ethanol is in the range of $50-90 \%$. The concentration of anthocyanins reached its maximum when ethanol concentration was $70 \%$. This is due to the use of ethanol solution in extracting agent can avoid other dissolution of water-soluble substances, more anthocyanins were dissolved out with the increase of ethanol concentration, however, less anthocyanins were dissolved out with the further increase of ethanol concentration, it is because the polarity of the solution decreases. When the polarity of the anthocyanins in close proximity to the solvent polarity, The extraction rate reached its maximum.

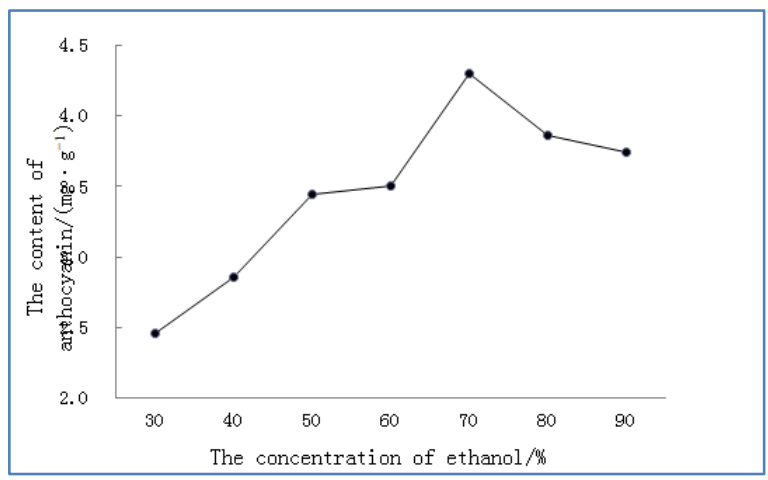

Fig-2: The influence of ethanol concentrations

\section{The influence of liquid-material ratio}

Figure 3 shows the changes of anthocyanin contents in the extraction under different liquid-solid ratio conditions. With the increase of the ratio, the anthocyanin content showed a wave-like rising trend, but when the ratio exceeded 70:1, the anthocyanin content did not increase but decreased. This is mainly because the dissolution of anthocyanin needs a certain amount of solvent, when the liquid is relatively low, the amount of solvent is less, and the anthocyanin in purple eggplant peel cannot be completely extracted by ethanol solution, so the optimal liquid to material ratio is $70: 1$.

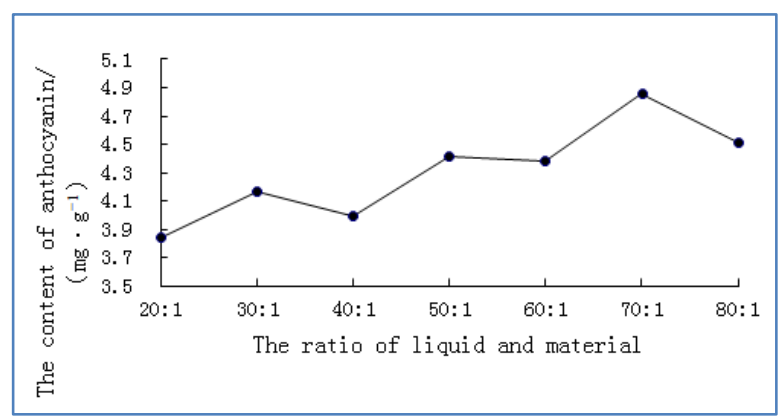

Fig-3: The effect of liquid to material ratio

\section{The influence of extraction time}

Figure 4 shows the changes of anthocyanin content under different extraction time. It can be seen that the content of anthocyanin increases gradually with the extension of time. When the extraction time is 180 min, the content of anthocyanin reaches the maximum, and then it decreases after reaching the peak. This is mainly because the dissolution of anthocyanin needs a certain time, so the anthocyanin in purple eggplant peel did not be completely extracted at relatively short time, which resulted a lower content of anthocyanin in the crude extract. However, the stability of anthocyanins may be affected if the time is too long. Therefore, the optimal extraction time is $180 \mathrm{~min}$.

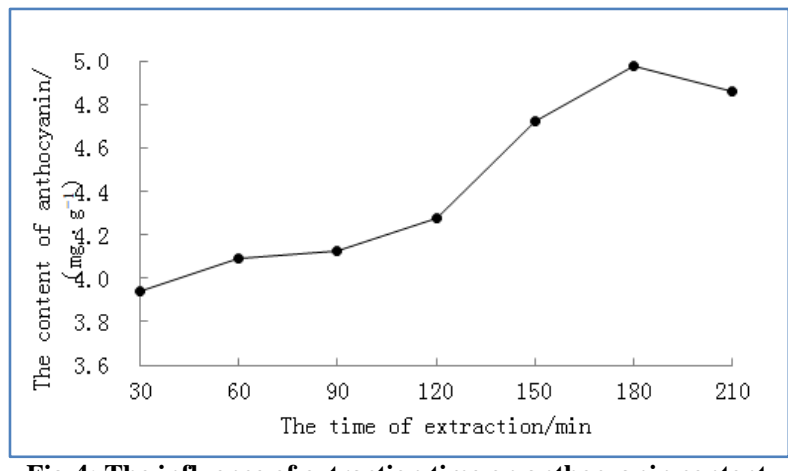

Fig-4: The influence of extraction time on anthocyanin content

\section{The influence of extraction temperature}

Figure 5 shows the changes of anthocyanin content at different extraction temperatures, and it can be seen that the extraction effect is better at low temperature. The maximum content of anthocyanin is at $40{ }^{\circ} \mathrm{C}$, the content of anthocyanin fells sharply when the temperature further raised, that is because the stability of anthocyanins changes as the change of temperature, the structure of the anthocyanins may be destroyed when the temperature is too high, the content of anthocyanins reduces as the degradation of anthocyanins, so the best extraction temperature is at 40 ${ }^{\circ} \mathrm{C}$.

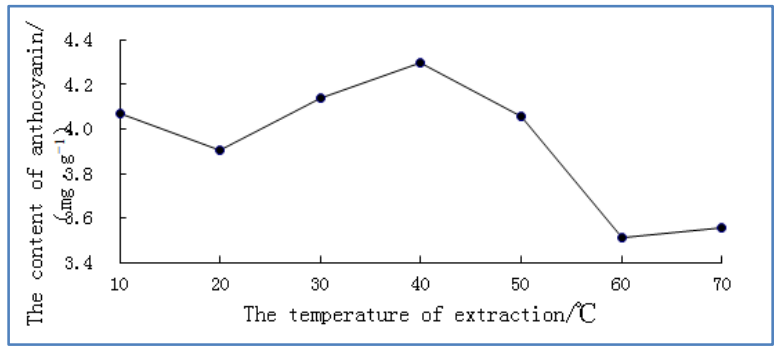

Fig-5: The effect of extraction temperature on anthocyanin content

\section{The experiment of quadratic orthogonal rotation combination}

\section{The design and results of experiment}

The factor level range was determined combining with the single-factor experiment. Ethanol concentration $\mathrm{x}_{1}(\%)$, liquid-solid ratio $\mathrm{x}_{2}\left(\mathrm{~mL} \cdot \mathrm{g}^{-1}\right)$, 
time $\mathrm{x}_{3}(\mathrm{~min})$ and temperature $\mathrm{x}_{4}\left({ }^{\circ} \mathrm{C}\right)$ were as the four factors. Study on optimization of extraction technology of anthocyanin extracted from purple eggplant peel by four factors and five levels quadric orthogonal rotation combination design.

Table-1: The level table of quadratic orthogonal rotation test factors

\begin{tabular}{|c|c|c|c|c|}
\hline \multirow{2}{*}{ Level } & \multicolumn{4}{|c|}{ Factors } \\
\cline { 2 - 5 } & $\begin{array}{c}\mathbf{X}_{\mathbf{1}} / \text { The } \\
\text { concentration of } \\
\text { ethanol (\%) }\end{array}$ & $\begin{array}{c}\mathbf{X}_{\mathbf{2}} / \text { The ratio of liquid } \\
\text { and material } \\
\left(\mathbf{m L} \cdot \mathbf{g}^{-1}\right)\end{array}$ & $\begin{array}{c}\mathbf{X}_{\mathbf{3}} / \text { The time of } \\
\text { extraction } \\
(\mathbf{m i n})\end{array}$ & $\begin{array}{c}\mathbf{X}_{\mathbf{4}} / \text { The temperature } \\
\left({ }^{\circ} \mathbf{C}\right)\end{array}$ \\
\hline-2 & 50 & $40: 1$ & 90 & 20 \\
\hline-1 & 60 & $50: 1$ & 120 & 30 \\
\hline 0 & 70 & $60: 1$ & 150 & 40 \\
\hline 1 & 80 & $70: 1$ & 180 & 50 \\
\hline 2 & 90 & $80: 1$ & 210 & 60 \\
\hline
\end{tabular}

Table-2: The experimental scheme and results of quadratic orthogonal rotation combination design

\begin{tabular}{|c|c|c|c|c|c|}
\hline \multirow[b]{2}{*}{ Number } & $\mathrm{X}_{1}$ & $\mathbf{X}_{2}$ & $\mathbf{X}_{\mathbf{3}}$ & $\mathbf{X}_{4}$ & \multirow{2}{*}{$\begin{array}{c}\text { Y The contentof } \\
\text { anthocyanin } \\
\left(\mathrm{mg} \cdot \mathrm{g}^{-1}\right)\end{array}$} \\
\hline & $\begin{array}{c}\text { The } \\
\text { concentration of } \\
\text { ethanol }(\%)\end{array}$ & $\begin{array}{c}\text { The ratio of liquid } \\
\text { and material } \\
\left(\mathrm{mL} \cdot \mathrm{g}^{-1}\right)\end{array}$ & $\begin{array}{c}\text { The time of } \\
\text { extraction } \\
\text { (min) }\end{array}$ & $\begin{array}{c}\text { The } \\
\text { temperature } \\
\left({ }^{\circ} \mathrm{C}\right)\end{array}$ & \\
\hline 1 & 1 & 1 & 1 & 1 & 3.1864 \\
\hline 2 & 1 & 1 & 1 & -1 & 3.5468 \\
\hline 3 & 1 & 1 & -1 & 1 & 3.4288 \\
\hline 4 & 1 & 1 & -1 & -1 & 3.7656 \\
\hline 5 & 1 & -1 & 1 & 1 & 3.2788 \\
\hline 6 & 1 & -1 & 1 & -1 & 3.7628 \\
\hline 7 & 1 & -1 & -1 & 1 & 3.2407 \\
\hline 8 & 1 & -1 & -1 & -1 & 3.6287 \\
\hline 9 & -1 & 1 & 1 & 1 & 2.7731 \\
\hline 10 & -1 & 1 & 1 & -1 & 3.2162 \\
\hline 11 & -1 & 1 & -1 & 1 & 2.7100 \\
\hline 12 & -1 & 1 & -1 & -1 & 3.0673 \\
\hline 13 & -1 & -1 & 1 & 1 & 2.7002 \\
\hline 14 & -1 & -1 & 1 & -1 & 3.3640 \\
\hline 15 & -1 & -1 & -1 & 1 & 2.7216 \\
\hline 16 & -1 & -1 & -1 & -1 & 2.8438 \\
\hline 17 & -2 & 0 & 0 & 0 & 2.9766 \\
\hline 18 & 2 & 0 & 0 & 0 & 3.7934 \\
\hline 19 & 0 & -2 & 0 & 0 & 2.8644 \\
\hline 20 & 0 & 2 & 0 & 0 & 3.0921 \\
\hline 21 & 0 & 0 & -2 & 0 & 4.0982 \\
\hline 22 & 0 & 0 & 2 & 0 & 3.8458 \\
\hline 23 & 0 & 0 & 0 & -2 & 4.0982 \\
\hline 24 & 0 & 0 & 0 & 2 & 3.4311 \\
\hline 25 & 0 & 0 & 0 & 0 & 4.1845 \\
\hline 26 & 0 & 0 & 0 & 0 & 4.0077 \\
\hline 27 & 0 & 0 & 0 & 0 & 4.0612 \\
\hline 28 & 0 & 0 & 0 & 0 & 4.1511 \\
\hline 29 & 0 & 0 & 0 & 0 & 4.0189 \\
\hline 30 & 0 & 0 & 0 & 0 & 4.1246 \\
\hline 31 & 0 & 0 & 0 & 0 & 4.0879 \\
\hline 32 & 0 & 0 & 0 & 0 & 4.0453 \\
\hline 33 & 0 & 0 & 0 & 0 & 2.8678 \\
\hline 34 & 0 & 0 & 0 & 0 & 3.2518 \\
\hline 35 & 0 & 0 & 0 & 0 & 2.9446 \\
\hline 36 & 0 & 0 & 0 & 0 & 3.3431 \\
\hline
\end{tabular}


According to the data in table 2, The relationship between $\mathrm{y}$ and $\mathrm{x} 1, \mathrm{x} 2, \mathrm{x} 3, \mathrm{x} 4$ was regression analysis by SPSS software was used for of $y$ and $\mathrm{x} 1, \mathrm{x} 2, \mathrm{x} 3$ and $\mathrm{x} 4$, The response equation of $\mathrm{y}$ and 1 $、 \mathrm{x}_{2} 、 \mathrm{x}_{3} 、 \mathrm{x}_{4}$ was established as the formula(1). $y=3.757+0.253 x_{1}+0.025 x_{2}-0.003 x_{3}-0.187 x_{4}-0.147 x_{1}^{2}-0.008 x_{1} x_{2}-0.062 x_{1} x_{3}+0.001 x_{1} x_{4}-$
$0.249 x_{2}^{2}-0.058 x_{2} x_{3}+0.010 x_{2} x_{4}-0.047 x_{3} x_{4}-0.052 x_{4}^{2}$

The data in table 2 were tested for the irregularity, the result is: $\mathrm{F}=0.7486<\mathrm{F} 0.05(10,11)$ $=2.98$, The test error is not significant. Then, variance analysis was conducted on response surface equation (1) to find that the equation reached a significant level. The specific data are shown in table 3. At last, the significance test of the regression coefficient of equation (1) was carried out, and it can be known that

$$
y=3.757+0.253 x_{1}-0.187 x_{4}-0.147 x_{1}^{2}-0.249 x_{2}^{2}
$$

The partial derivative of $\mathrm{y}$ to $\mathrm{x} 1, \mathrm{x} 2, \mathrm{x} 4$ was respectively obtained through the equation (2), and make them to zero, the best extraction technology conditions was obtained by calculating the equations, it is concluded that $\mathrm{x} 1=0.8605, \mathrm{x} 2=0, \mathrm{x} 4=2$, because the $\mathrm{x} 3$ is not significant, the zero level was selected, the order of influence of four factors on the extraction of anthocyanins from eggplant pee is as follows: ethanol concentration $>$ extraction temperature > ratio of liquid-solid $>$ extraction time. The non-significant terms were eliminated at the significant level of 0.05 , and the optimized response equation was obtained as the formula (2). namely, the ethanol concentration is $78.6 \%$, the ratio of liquid and material is $60: 1\left(\mathrm{~mL} \cdot \mathrm{g}^{-1}\right)$, the extraction temperature is $20^{\circ} \mathrm{C}$, the extraction time is $150 \mathrm{~min}$, the content of anthocyanin is up to $4.2399 \mathrm{mg} \cdot \mathrm{g}^{-1}$ under this conditions.

Table-3: The analysis of variance and test of irregularity of the quadratic orthogonal rotating combinatorial

\begin{tabular}{|l|c|c|c|c|c|}
\hline design test \\
\hline Sources of variation & SS & df & ms & $\boldsymbol{F}$ & $\boldsymbol{P}$ \\
\hline Returning & 435.1871 & 14 & 31.0848 & 151.8172 & $<0.0001$ \\
\hline Remaining & 4.2998 & 21 & 0.2048 & & \\
\hline Error & 2.7670 & 11 & 0.2515 & & \\
\hline Loss of quasi & 1.5328 & 10 & 0.1533 & 0.7486 & 0.3967 \\
\hline Tolal & 443.7867 & 35 & & & \\
\hline
\end{tabular}

\section{The analysis of interaction effect}

Any two of ethanol concentration $\left(x_{1}\right.$, the ratio of liquid and material (x2) and the extracting temperature $(\mathrm{x} 4)$ were interactive analysed to ascertain the effect of the anthocyanin content (y) by mathematica software, the image below are the response surface and contour plot of interacting factors, It can visually reflect the influence of interaction effect on the content of anthocyanins [12].

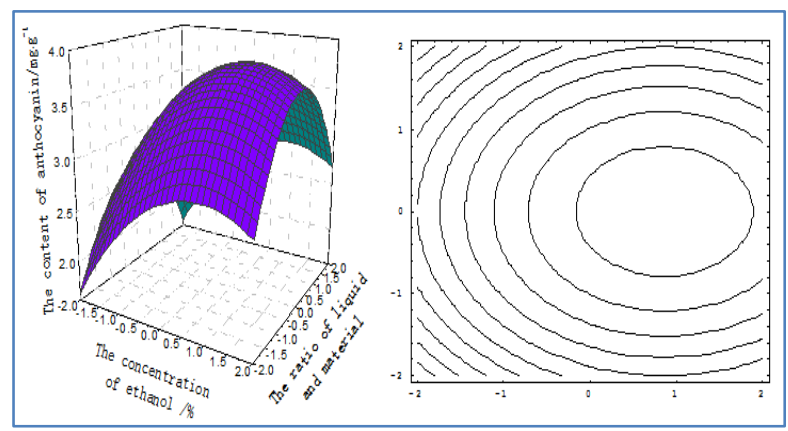

Fig-6: The response surface and contour map of $y=f\left(x_{1}, x_{2}\right)$

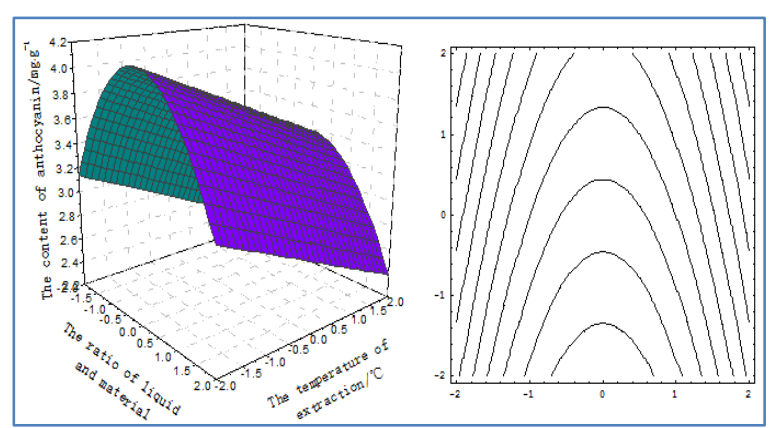

Fig-7: The response surface and contour map of $y=f\left(x_{2}, x_{4}\right)$

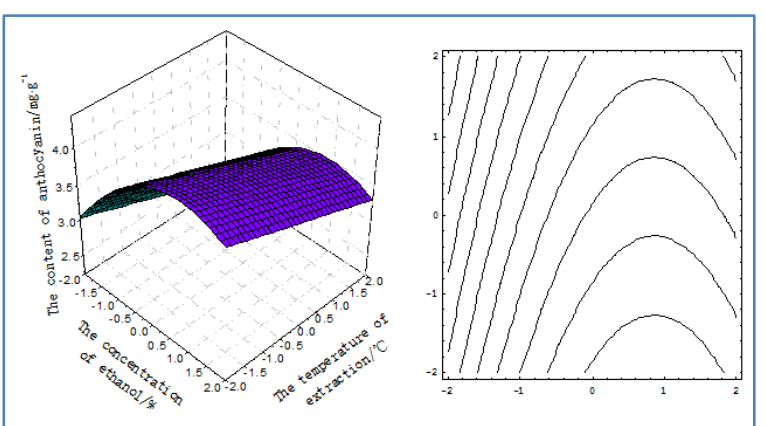

Fig-8: The response surface and contour map of $y=f\left(x_{1}, x_{4}\right)$ 
Figure 6 shows that the content of anthocyanin in the crude extract increased with the increase of ethanol concentration and the rate of liquid and material, when the concentration of ethanol and the ratio of liquid and material reaches a certain amount, the content of anthocyanin in the crude extract declined, the contour displayed as elliptic, the interaction effect of ethanol concentration on the rate of liquid and material are significant. It can be seen in figure 6 , the ethanol concentration on the side of the contour is dense, it illustrates that the effect of ethanol concentration is bigger than the rate of liquid and materials on the extraction of anthocyanin.

It can be seen in figure 7 that the content of anthocyanin in the crude extract gradually increased with the decrease of extraction temperature and the increase of the ratio of liquid-material, and when the temperature of extraction and the ratio of liquidmaterial reached a certain amount, the content of anthocyanin decreased.

It can be seen in figure 8 that the content of anthocyanin in the crude extract gradually increased with the increase of ethanol concentration and the decrease of extraction temperature, When the ethanol concentration and extraction temperature reached a certain level, the content of anthocyanin decreased, but the trend of increase and decline is gentle, indicating that the interaction effect between the concentration of ethanol and the temperature of extraction is not significant.

\section{The verification experiment}

The anthocyanin in purple eggplant peel was extracted under the optimized conditions. The anthocyanin content was $4.1758 \mathrm{mg} \cdot \mathrm{g}-1$, it was slightly lower than that of the predicted value.

\section{CONCLUSIONS}

As a kind of natural pigment, anthocyanin has many physiological functions. More and more attention has been paid to its development and utilization. The results showed that the anthocyanin content in purple eggplant peel was rich. Based on the single factor experiment, the quadratic orthogonal rotating combination design experiment was carried out, and the regression model was established to optimize the extraction process of anthocyanins in purple eggplant skin. The results showed that each factor had different influence on the extraction effect of anthocyanins. The best extraction technology conditions were obtained by regression model: the ethanol concentration is $78.6 \%$, the rate of liquid and material is $60: 1\left(\mathrm{~mL} \cdot \mathrm{g}^{-1}\right)$, the extracting temperature is $20^{\circ} \mathrm{C}$, the extraction time is
150 min. Under this condition, the extraction of anthocyanins in purple peel was carried on, the measured result is $4.1758 \mathrm{mg}$, it is close to the forecast result $\left(4.2399 \mathrm{mg} \cdot \mathrm{g}^{-1}\right)$, that illustrates that this method optimized extraction process of anthocyanins in purple peel is reliable, and it has a certain practical guiding significance.

\section{REFERENCES}

1. Cunming, X.U., Gaoyang, P., \& Ting, 1. I. (2013). Research progress on the physiological activity of anthocyanins [J]. China food additives, 3:205-210.

2. Dequn, S., Xianjun, M., Chenyang, W. (2013). Determination of anthocyanins in blueberry by $\mathrm{pH}$ differential method $[\mathrm{J}]$. Journal of Shenyang agricultural university, 43(2):231-233.

3. Lei, W., Jiafei, L.V., Zhizhou, L.I. (2010). Research progress in extraction, separation and purification of anthocyanins $[\mathrm{J}]$. Chemical science and technology market, 33(2):14-17.

4. Rihan, W.U, Yanru, F.U., Zhang, Y.E. (2016). Research status and development trend of natural food coloring-anthocyanin $[\mathrm{J}]$. Modern food, 16:35-36.

5. Lijuan, D.U, Qingping, W., Jinliang, S. (2016). Optimization of response surface method for determination of anthocyanins in lycium barbarum[J]. Chinese food and nutrition, 22(6):5054.

6. Lihua, P., Jianfei, W., Xingqian, Y.E. (2014). Extraction of blueberry anthocyanin and its immunoregulatory activity $[\mathrm{J}]$, Food science, 35(2):81-86.

7. Jinqiu, W. (2016). Extraction, purification and application of anthocyanins in purple eggplant skin [D]. Harbin: Harbin University of commerce.

8. Shaozhu, F. (2017). Status quo of eggplant industry in China and progress of eggplant character evaluation methods [J]. Jilin vegetables, 6:43-44.

9. Lingling, L.U., Xuefeng, F., Wei, L.I. (2008). Advances in research on anthocyanins in eggplant [J]. Molecular plant breeding, 16(15):5065-5071.

10. Fuleki, T., \& Francis, F. J. (1968). Extraction and determination of total anthocyanin in cranberries. $J$ Food Sci, 33(1), 72-77.

11. Song, Y., Beibei, Z., Jianghua, C. (2012). Optimization of extraction process of anthocyanin by quadratic orthogonal rotating combination design [J]. Food science, 33(8):33-38.

12. Yang, Z., \& Zhai, W. (2010). Optimization of microwave-assisted extraction of anthocyanins from purple corn (Zea mays L.) cob and identification with HPLC-MS. Innovative food science \& emerging technologies, 11(3), 470-476. 